\title{
Spectroscopic investigation of a FRET molecular beacon containing two fluorophores for probing DNA/RNA sequences $\dagger$
}

\author{
Steffen Jockusch, ${ }^{a}$ Angel A. Martí, ${ }^{a}$ Nicholas J. Turro, ${ }^{* a, b}$ Zengmin $\mathrm{Li},{ }^{b, c}$ Xiaoxu Li, ${ }^{c}$ Jingyue Ju, ${ }^{b, c}$ \\ Nathan Stevens ${ }^{d}$ and Daniel L. Akins ${ }^{d}$
}

Received 9th January 2006, Accepted 8th March 2006

First published as an Advance Article on the web 21st March 2006

DOI: $10.1039 / \mathrm{b600213g}$

We report the design, synthesis, and characterization of a molecular beacon (MB) consisting of two fluorescent dyes (Alexa 488 and RedX) for DNA and RNA analysis. In the absence of the target DNA or RNA the MB is in its stem-closed form and shows efficient energy transfer from the donor (Alexa) to the acceptor (RedX), generating mostly fluorescence from RedX. In the presence of the complementary target DNA the MB opened efficiently, hybridizes with the target DNA, and energy transfer is blocked in the stem-open form. This attachment to the target generates a fluorescence signature, which is clearly distinguishable from the fluorescence signature of the stem-closed form, allowing for ratiometric analysis of the fluorescence signal. In addition to steady-state fluorescence analysis, time resolved fluorescence (ps time range) and fluorescence depolarization studies were performed. We show that fluorescence lifetime and fluorescence depolarization measurements are useful analytical tools to optimize the MB design.

\section{Introduction}

Molecular beacons (MB) have the potential to become powerful tools for detecting target DNA and RNA in living cells. ${ }^{1}$ In general, a $\mathrm{MB}$ is a single stranded oligonucleotide that forms a stem-loop structure (Scheme 1 and 2)., ${ }^{2,3}$ The loop is complementary to the target sequence while the stem is labeled with a fluorescence sensor. "Classical" MBs possess a structure consisting of a fluorophore (sensor) and a quencher attached to opposite ends of a stem (Scheme 1, left). ${ }^{1,45}$ In the stem-closed form of the MB the fluorescence of the sensor is quenched due to the close proximity of the quencher. In the presence of the target sequence of RNA or DNA the MB opens to form a double stranded structure. In this stem-open form, the fluorescent sensor and the quencher are separated and strong fluorescence of the sensor is observed. The sensitivity of MBs as an analytical probe is determined mainly by two parameters: any residual fluorescence when the $\mathrm{MB}$ is in the stem-closed form ("false positive signals" due to incomplete quenching ${ }^{6}$ or adventitious impurities) and the fluorescence of the sensor when it is in the stem-open form. Ideally, the fluorophore should be quenched completely by the quencher through fluorescence resonance energy transfer (FRET) in the stem-closed form. In reality, however, the residual fluorescence varies greatly, largely due to factors such as the rigidity or

${ }^{a}$ Departments of Chemistry, Columbia University, New York, NY, 10027

${ }^{b}$ Departments of Chemical Engineering, Columbia University, New York, NY, 10027

${ }^{c}$ Columbia Genome Center, Columbia University College of Physicians and Surgeons, New York, NY, 10032

dDepartment of Chemistry, City College, City University of New York, NY, 10031

$\dagger$ Electronic supplementary information (ESI) available: Details of the synthesis of the MB, UV-Vis absorption spectra of the MBs (Fig. S1), fluorescence spectra of Alexa-MB and MB-RedX (Fig. S2), target DNA concentration dependence (Fig. S3), and no interaction of the MB with non-complementary target DNA (Fig. S4). See DOI: 10.1039/b600213g
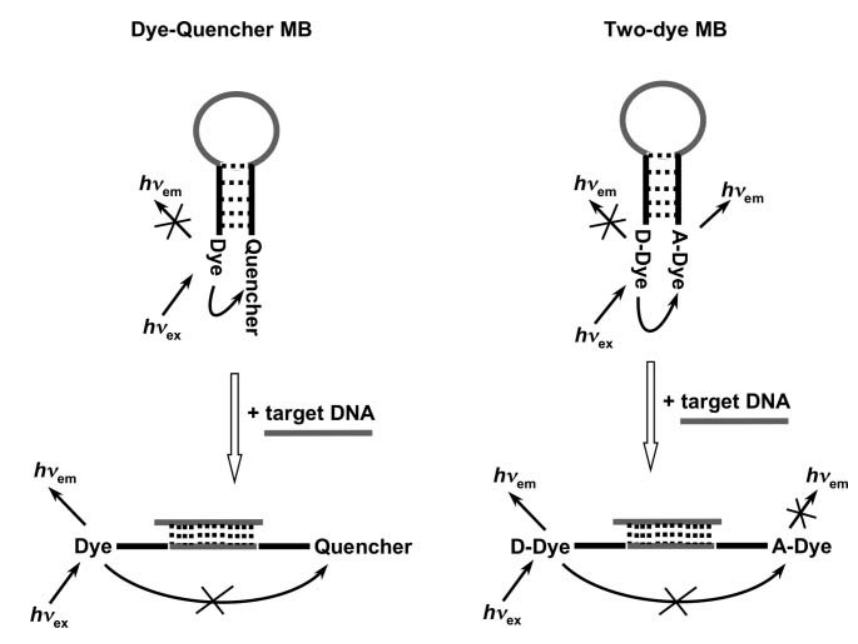

Scheme 1 Schematic representation of a dye-quencher MB and two-dye MB.

flexibility and length of the loop, ${ }^{7}$ the purity of the MB, the selection of fluorophore/quencher properties, and the way the fluorophore/quencher groups are attached to the oligonucleotide. ${ }^{8}$ Any residual fluorescence when the $\mathrm{MB}$ is in its closed form greatly limits the detection sensitivity. In addition, MBs of the fluorophore-quencher type can rarely be used to detect unknown targets quantitatively.

In an effort to overcome these limitations of the classical MB, a new type of $\mathrm{MB}$ has been reported which uses a quencher that itself is fluorescent (Scheme 1, right). ${ }^{9}$ This MB contains two fluorophores, a donor dye (coumarin) and an acceptor dye (6carboxyfluorescein), which are covalently attached at opposite ends of the stem. In the stem-closed form the coumarin fluorescence is quenched by the acceptor dye, 6-carboxyfluorescein, through FRET, generating fluorescence of the acceptor dye, which is at a different wavelength than the fluorescence of the donor 


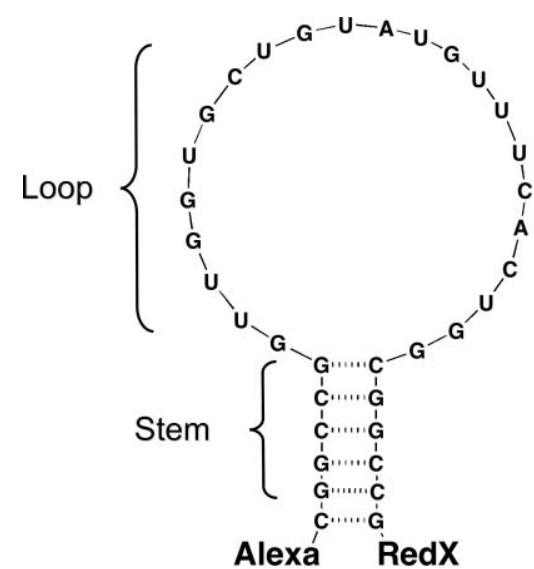

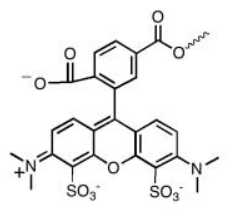

Alexa 488

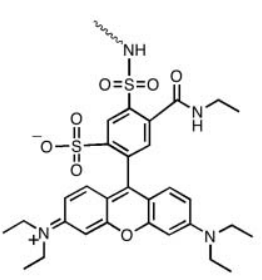

RedX

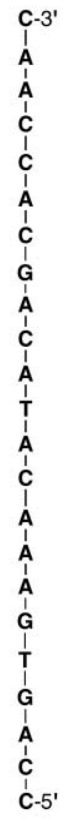

target DNA
Scheme 2 Structure of the two-dye MB, Alexa-MB-RedX, and the target DNA. The two dyes, Alexa and RedX, are covalently linked at the $5^{\prime}$ and $3^{\prime}$ position, respectively.

dye. In the stem-open form FRET is negligible and mainly the fluorescence of coumarin is observed. ${ }^{9}$ It was demonstrated that this two-dye MB shows a higher sensitivity than conventional dyequencher MBs. Moreover, the change in fluorescence wavelength upon detection of target allows for a ratiometric fluorescence analysis. Ratiometric analysis is usually superior compared to intensity based measurements, ${ }^{9,10}$ such as in conventional dyequencher MB. Furthermore, ratiometric analysis is less dependent on $\mathrm{MB}$ concentration fluctuations, photobleaching of the dye, optical geometry and other environmental factors. ${ }^{9}$ Other MBs that employ pyrenes as fluorophores for ratiometric analysis have been reported. These MBs switch their fluorescence wavelength by exploiting monomer or excimer photoluminescence in the stemopen and stem-closed form, respectively. ${ }^{11,12}$

In this paper we report on a $\mathrm{MB}$, which contains two fluorophores, the donor dye Alexa 488 and the acceptor dye RedX, attached at opposite ends of the oligonucleotide (Scheme 2). The selected stem consists of six $\mathrm{G}-\mathrm{C}$ base pairs, because $\mathrm{G}-$ $\mathrm{C}$ base pairs are known to be more stable than $\mathrm{A}-\mathrm{U}$ base pairs. ${ }^{13}$ This higher stability of the stem reduces the non-specific opening of the $\mathrm{MB}$ in the absence of target. The loop sequence was selected based on our interest to study sensorin mRNA in neurons. We selected as a target the 271-293 region of sensorin mRNA, which is present in the sensory neurons of the mollusk Aplysia Californica. ${ }^{14}$ This loop sequence presents little secondary structure. Therefore, competition with the sensorin sequence due to self-complementarity is not expected. The light absorber, the donor dye Alexa, ${ }^{15}$ was selected based on its favorable excitation wavelength (488 nm), which can be provided by an argon-ion laser available in most fluorescence microscopes. In addition, compared to previously reported two-color MBs, ${ }^{9,11,12}$ which used UV absorbing chromophores, the longer excitation wavelength, since it avoids direct excitation of biological molecules, is more attractive for biological applications.

The MB contains modified 2' $O$-methyloligoribonucleotides that are known to hybridize more efficiently, have higher stability, and are more resistant to enzymatic degradation when compared to unmodified deoxyribonucleotides. ${ }^{16-18}$

\section{Experimental}

\section{Synthesis of MB}

Alexa-MB-RedX was prepared by solid-phase phosphoramidite chemistry on a DNA synthesizer (Expedite 8909; Applied Biosystems) and solution coupling chemistry. Instead of the regular phosphoramidite, the modified 2'-methoxy $N$ phosphoramidite was used. The monomers, 5' amino-modifier C6 phosphoramidite and 3'-amino-modifier C7 CPG were purchased from Glen Research (Sterling, VA), and Alexa 488 and RedX NHS were purchased from Molecular Probes (Eugene, OR). After the deprotection under mild conditions (concentrated $\mathrm{NH}_{4} \mathrm{OH}$ at $40{ }^{\circ} \mathrm{C}, 17 \mathrm{~h}$ ), the intermediate $5^{\prime}$-MMT protected amino and 3 -amino-RNA was obtained. Then, the product was coupled with RedX NHS in DMSO in $0.1 \mathrm{M}$ sodium tetraborate buffer $\left(\mathrm{pH}=8.5\right.$ ) or $0.1 \mathrm{M} \mathrm{NaHCO}_{3}-\mathrm{Na}_{2} \mathrm{CO}_{3}$ buffer $(\mathrm{pH}=8-9)$ at room temperature overnight. The coupled product was deprotected by treating with $20 \% \mathrm{HAcO}$ for $1 \mathrm{~h}$ at room temperature, extracted three times with ethyl acetate, and the aqueous layer was dried to yield $5^{\prime}-\mathrm{NH}_{2}-\mathrm{RNA}-\mathrm{NH}-\mathrm{RedX}-3^{\prime}$. The residue was purified by gel electrophoresis (6 M urea-TBE, $20 \%$ acrylamide gel). The purified fraction was coupled with Alexa 488 NHS in $0.1 \mathrm{M}$ of sodium tetraborate or $\mathrm{NaHCO}_{3}-\mathrm{NaCO}_{3}$ buffer ( $\mathrm{pH}=8-9$ ) overnight to form the desired Alexa-MB-RedX product (Scheme 2). The crude compound was further purified by HPLC using a Waters system consisting of a $7725 \mathrm{i}$ injector, 600 controller, and a 960 photodiode array detector. An Xterra MS C18 $(4.6 \times 50 \mathrm{~mm})$ column was used. The elution was performed over $90 \mathrm{~min}$ at a flow rate of $0.5 \mathrm{ml} \mathrm{min} \mathrm{m}^{-1}$ and a fixed temperature of $60{ }^{\circ} \mathrm{C}$, using a linear gradient (12-34.5\%) of methanol in a buffer containing $8.6 \mathrm{mM}$ triethylamine and $100 \mathrm{mM}$ hexafluoroisopropyl alcohol $(\mathrm{pH}=8.1)$. The purified MB was identified by MALDI-TOF [13291 (Found), 13291(Calcd)] using a Voyager DE mass spectrometer (Applied Biosystems). Purity 95\%.

The single dye MBs, Alexa-MB and MB-RedX, were synthesized analogues to Alexa-MB-RedX.

\section{Synthesis of the target DNA}

The target DNA (Scheme 2) was synthesized by standard solidphase phosphoramidite chemistry on a DNA synthesizer (Expetide 8909; Applied Biosystems). The obtained crude product was deprotected by concentrated $\mathrm{NH}_{4} \mathrm{OH}$, purified by OPC (Oligonucleotide Purification Cartridge, Applied Biosystems, Foster, CA) and RP-HPLC, and was characterized by MALDI-TOF: 6975 (Calcd), 6978 (Found) ( 95\% purity).

\section{Steady-state fluorescence and polarization measurement}

Fluorescence emission spectra and polarization values were recorded at room temperature on a SPEX Fluorolog-3 spectrometer FL3-22 (J. Y. Horiba, Edison, NJ) using quartz cuvettes with 
path length of $4 \mathrm{~mm}$ and Glan Thompson polarizers, respectively. The spectra were corrected for detector response and grating efficiency according to the correction factor file pertaining the instrument. The polarization values $(P)$ for the individual dye components and the energy transfer components were determined by exciting one dye with linear polarized light and analyzing the depolarization of the fluorescence at different wavelengths for the two fluorophores.

\section{Time-resolved fluorescence measurement}

Time-dependent emission measurements utilized a Hamamatsu streak camera, Model C4334, optically coupled to a chargecoupled-device (CCD) array detector. This system allowed the measurements of both the emission decay and the time resolved emission spectrum. For this latter study a Chromex 205i imaging spectrometer was used. The excitation source for these studies was an all-solid state laser system from Spectra Physics, which incorporates the following components: a diode-pumped Millennia V-P laser for exciting mode-locked lasing from a Tsunami (Model 3941M1S) Ti-sapphire laser, which in turn was amplified by a Spitfire regenerative amplifier with a Merlin regenerative pump source. Tunable, femtosecond radiation was acquired through the use of an optical parametric amplifier (OPA-800P) in combination with harmonic generation and sum-difference packages. The tunable laser pulses were of $c a$. $150 \mathrm{fs}$ in duration at a repetition rate of 1 $\mathrm{kHz}$. For the present study, $488 \mathrm{~nm}$ pulses were used to excite the oligonucleotide system. The ultimate time resolution that we have been able to attain with this system, using Hamamatsu U4290 fluorescence analysis software, was estimated to be $\sim 10$ ps.

\section{Results and discussion}

Steady-state fluorescence analysis of Alexa-MB-RedX was performed at an excitation wavelength of $488 \mathrm{~nm}$. Fig. 1a shows the fluorescence spectrum of the stem-closed form, where the peak at

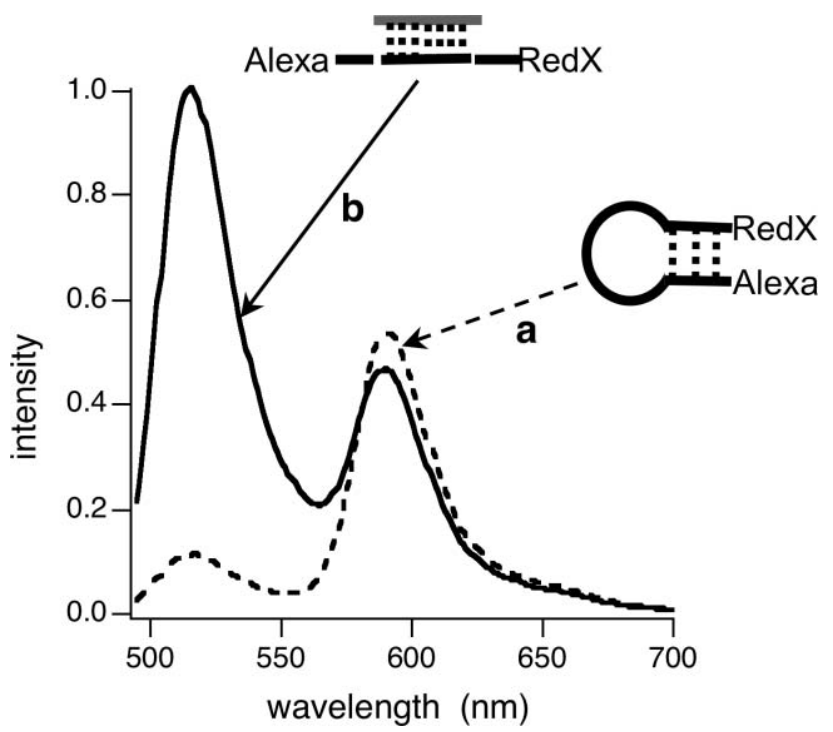

Fig. 1 Fluorescence spectra after excitation of Alexa-MB-RedX $(1 \mu \mathrm{M})$ in aqueous buffer solutions (20 mM Tris, $60 \mathrm{mM} \mathrm{MgCl}_{2}$ ), $500 \mathrm{mM}$ $\mathrm{NaCl}, \mathrm{pH}=7.0$, at $488 \mathrm{~nm}$ in the absence (a) and presence (b) of target DNA $(10 \mu \mathrm{M})$.
$515 \mathrm{~nm}$ corresponds to the fluorescence of the donor dye, Alexa, and the peak at $592 \mathrm{~nm}$ corresponds to the fluorescence of the acceptor dye, RedX. A ratio between the intensities of the donor $\left(I_{\text {Alexa }}^{\text {closed }}\right)$ and the acceptor dye $\left(I_{\text {RedX }}^{\text {closed }}\right)$ fluorescences of $0.2: 1$ was observed for the stem-closed form of the MB. The significantly higher fluorescence intensity of the acceptor dye compared to the donor dye is consistent with efficient energy transfer in the stemclosed form. Upon addition of the complementary target ssDNA the fluorescence signature changed significantly (Fig. 1b). A ratio of the fluorescence intensities between the donor $\left(I_{\text {Alexa }}^{\text {open }}\right)$ and the acceptor dye $\left(I_{\mathrm{RedX}}^{\text {open }}\right)$ of $2.1: 1$ was observed, which is consistent with diminished energy transfer in the stem-open form. The change in fluorescence ratio upon addition of the target DNA corresponds to a contrast ratio (eqn (1)) of approximately 10.

$$
\text { contrast ratio }=\frac{I_{\text {Alexa }}^{\text {open }} / I_{\text {RedX }}^{\text {open }}}{I_{\text {Alexa }}^{\text {closed }} / I_{\text {RedX }}^{\text {closed }}}=\frac{2.1}{0.2}=10.5 \mathrm{a}
$$

For practical applications of the $\mathrm{MB}$, a high contrast ratio is desired to detect target DNA or RNA sequences. However, the contrast ratio is reduced by the residual emission of the donor dye (e.g. Alexa) in the stem-closed form and the residual emission of the acceptor dye (e.g. RedX) in the stem-open form.

To investigate the residual emissions in detail and ultimately improve the MB design, time-resolved fluorescence experiments were performed. Laser excitation at $488 \mathrm{~nm}$ (150 fs pulse width) of the stem-closed form yielded time-resolved fluorescence spectra and kinetic decay traces using a streak-camera. Fig. 2 shows the fluorescence decay traces of the donor dye (Alexa) $(a, b)$ and the acceptor dye $(\operatorname{RedX})(\mathrm{c}, \mathrm{d})$ at a short $(\mathrm{b}, \mathrm{d})$ and a long $(\mathrm{a}, \mathrm{c})$ observation time window. Excitation of Alexa generated a fluorescence, which decayed multi-exponentially (see Table 1). The fastest component of the decay $\left(\tau_{3}<50 \mathrm{ps}\right)$ (Fig. 2b), within our instruments response, is attributed to the energy transfer quenching of Alexa by RedX (Fig. 2f). The longer components $\left(\tau_{2}\right.$ and $\tau_{1}$ ) contribute to most of the fluorescence $(\sim 98 \%)$. To ascertain if these slow decay components are caused by rate limiting energy transfer quenching (slow generation of excited states of RedX) or are caused by defective MBs, where energy transfer quenching is hindered, time-resolved fluorescence experiments were also performed on a stem-closed MB which contains only Alexa, but no RedX (Alexa-MB). Fig. 3c shows that the fluorescence decay of Alexa-MB in the stem-closed form is very similar to the slow component decay of Alexa-MB-RedX (Fig. 3a). In addition, a comparison of the fluorescence lifetimes reveals that $\tau_{2}$ and $\tau_{1}$ as well as the intensity contributions are similar for both MBs (Table 1). Therefore, we conclude that the slow decay components ( $\tau_{2}$ and $\tau_{1}$ ) of the "stem-closed" Alexa-MB-RedX are not caused by slow energy transfer and does not generate excited states of RedX. We interpret our observations as suggesting one or more of the following: (i) energy transfer in a small fraction of the sample is hindered, probably because some of the MBs opened without the presence of target; (ii) some conformations of the stem-closed MB sterically hinder energy transfer; and (iii) some of the MBs do not contain a quencher. We note further that the fluorescence decays of Alexa in the stem-open MBs, i.e., Alexa-MB-RedX (Fig. 3b) and Alexa-MB (d), as well as that for the stem-closed Alexa-MB (c) are similar as expected (see also Table 1). 

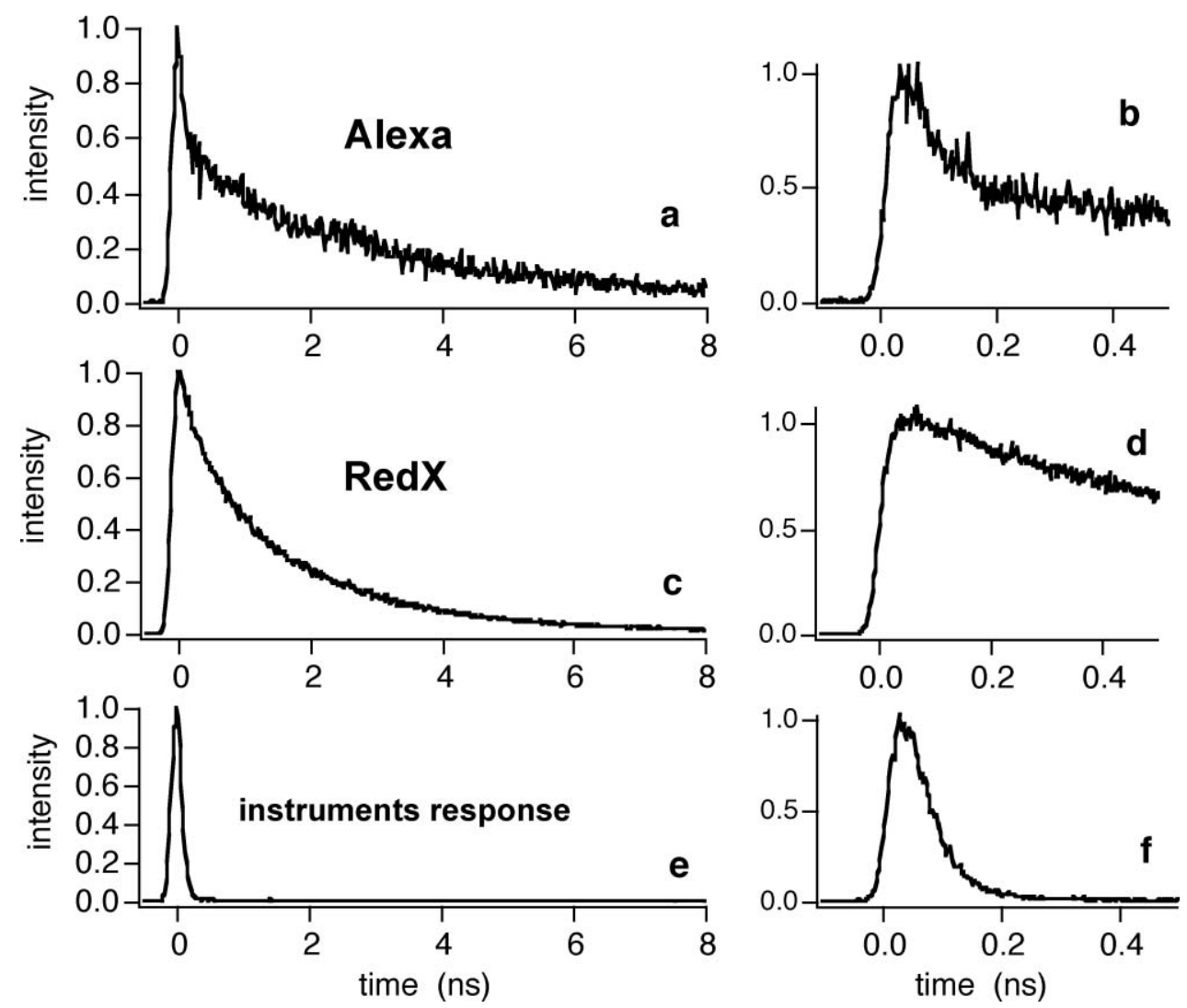

Fig. 2 Fluorescence decay of Alexa (a,b) monitored from 510 to $530 \mathrm{~nm}$ and RedX (c,d) monitored at 580 to $620 \mathrm{~nm}$ after laser excitation of Alexa at $488 \mathrm{~nm}(150 \mathrm{fs}$ pulses) in aqueous buffer solutions of Alexa-MB-RedX $(1 \mu \mathrm{M})$. The temporal instruments response is shown in (e,f).

Table 1 Photophysical parameters of stem-closed (absence of target) and stem-open (presence of target; $10 \mu \mathrm{M})$ of $\mathrm{MBs}(1 \mu \mathrm{M})$

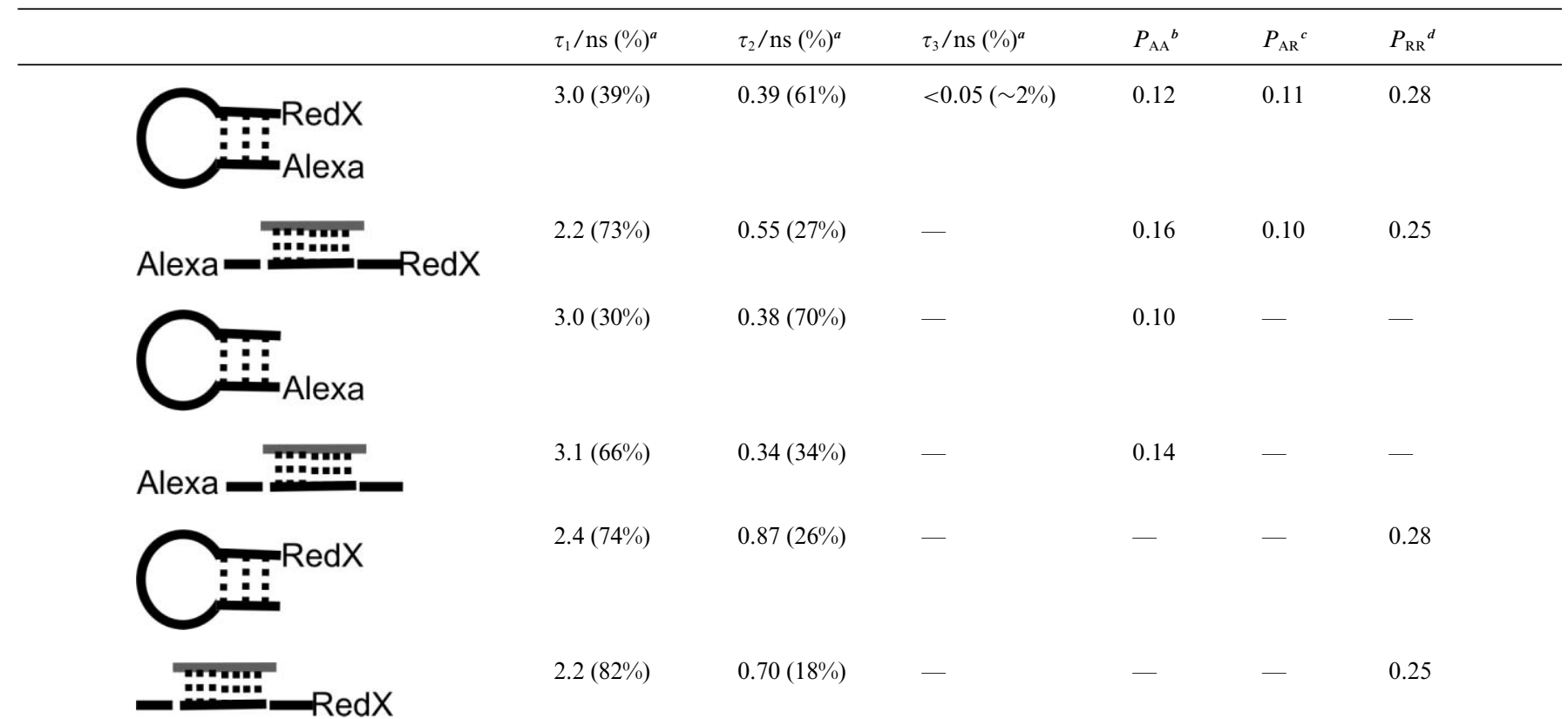

${ }^{a}$ Fluorescence lifetimes $(\tau)$ and corresponding fluorescence contribution $(\%) .{ }^{b}$ Polarization values $\left(P_{\mathrm{AA}}\right)$ for excitation of Alexa and emission of Alexa. ${ }^{c}$ Polarization values $\left(P_{\mathrm{AR}}\right)$ for excitation of Alexa and emission of RedX. ${ }^{d}$ Polarization values $\left(P_{\mathrm{RR}}\right)$ for excitation of RedX and emission of RedX. 


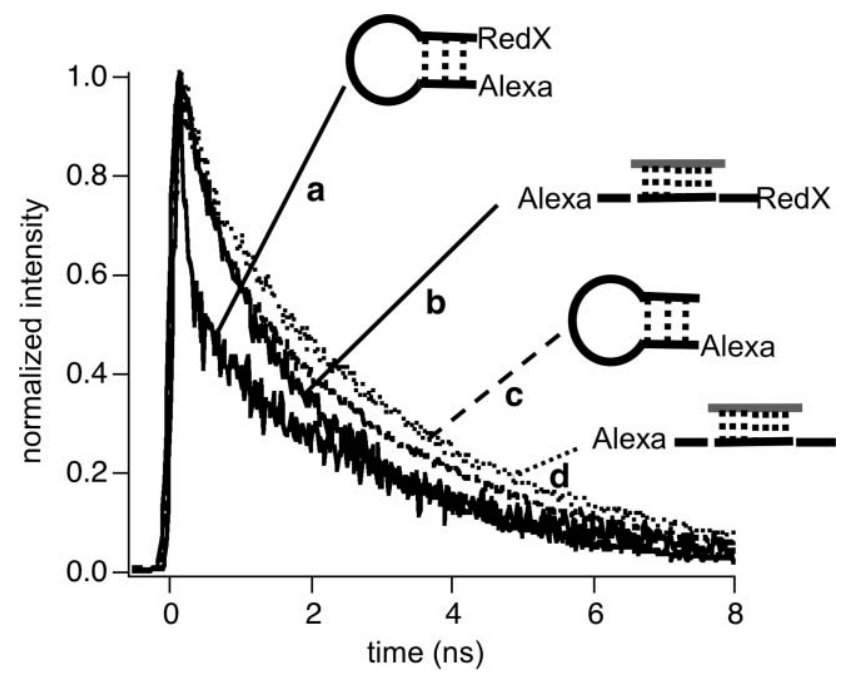

Fig. 3 Fluorescence decay of Alexa monitored from 510 to $530 \mathrm{~nm}$ after laser excitation of Alexa at $488 \mathrm{~nm}$ (150 fs pulses) in aqueous buffer solutions of Alexa-MB-RedX $(a, b)$ and Alexa-MB $(c, d)$ in the absence $(\mathrm{a}, \mathrm{c})$ and presence $(\mathrm{b}, \mathrm{d})$ of target DNA $(10 \mu \mathrm{M})$.

Steady-state fluorescence spectra of the stem-closed AlexaMB (absence of target) and stem-open Alexa-MB (presence of target) showed strong fluorescence of Alexa. However, the fluorescence intensity of the stem-open form (presence of target) increased approximately $35 \%$ compared to the stem-closed form (see electronic supplementary information (ESI), Fig. S2 $\dagger$ ). This is consistent with time-resolved fluorescence experiments (Fig. 3), where the decay of Alexa in the stem-closed form of Alexa-MB (c) is slightly faster than in the stem-open form (d). This quenching effect of Alexa in the stem-closed form is probably caused by guanosine residues. Because Alexa is covalently attached to $\mathrm{dC}$, in the stem-closed form $\mathrm{dG}$ is in close proximity due to hybridization with $\mathrm{dC}$ (Scheme 2) and probably causing some quenching. In the stem-open form the guanosine residue is separated from Alexa and an approximately $35 \%$ stronger fluorescence is observed (see ESI, Fig. S2 $\dagger$ ). Similar quenching effects have been reported for fluorescent dye linked oligonucleotides. ${ }^{19-21}$

The fluorescence lifetime of the acceptor dye, RedX, was examined. Fig. 4 shows that the fluorescence decays of RedX in the stem-open Alexa-MB-RedX (b), stem-open MB-RedX (d), and stem-closed MB-RedX (c) are very similar (see also Table 1). However, the fluorescence of RedX decayed slightly faster in the stem-closed Alexa-MB-RedX (a). Probably some quenching of excited states of RedX by Alexa occurred due to the close proximity of both dyes. Although both dyes, Alexa and RedX, are known to have single exponential decays in free solution, ${ }^{22}$ it is frequently reported that dyes covalently bound to DNA or RNA show multi-exponential fluorescence decays due to inhomogeneity and nucleotide interactions. ${ }^{23}$

Fluorescence depolarization studies provided further information on the photophysics of the MBs. Table 1 shows the polarization values $(P)$ for the individual fluorophores and energy transfer components. The $P$ values were determined by exciting one fluorophore with linearly polarized light and analyzing the depolarization of the fluorescence at different wavelength corresponding to the individual fluorophores. A fluorophore can lose its polarization by rotation during its fluorescence lifetime. ${ }^{24}$

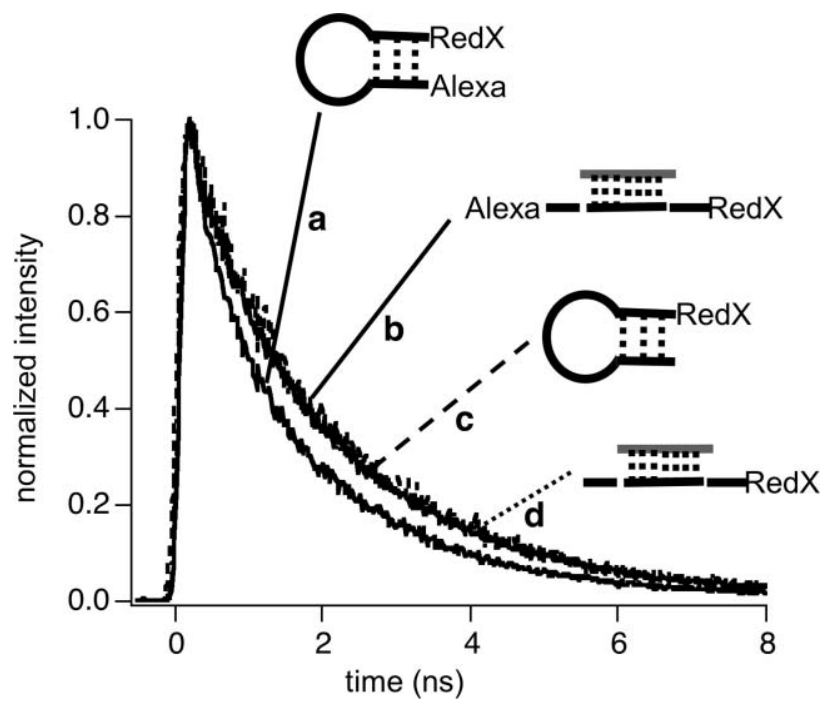

Fig. 4 Fluorescence decay of RedX monitored from 580 to $620 \mathrm{~nm}$ after laser excitation at $488 \mathrm{~nm}$ (150 fs pulses) in aqueous buffer solutions of Alexa-MB-RedX (a,b) and Alexa-MB (c,d) in the absence $(a, c)$ and presence $(\mathrm{b}, \mathrm{d})$ of target DNA $(10 \mu \mathrm{M})$.

The similar polarization values of Alexa $\left(P_{\mathrm{AA}}\right)$ for the MBs are in agreement with their similar fluorescence lifetimes (Fig. 3). Fluorescence polarization transfer from Alexa to RedX was observed in the stem-closed Alexa-MB-RedX $\left(P_{\mathrm{AR}}=0.11\right)$. This indicates efficient fluorescence energy transfer. The polarization values of RedX $\left(\mathrm{P}_{\mathrm{RR}}\right)$ for all four samples (stem-closed and stemopen Alexa-MB-RedX and MB-RedX) are very similar, which is consistent with the similar fluorescence lifetimes (Fig. 4).

To investigate the photophysical properties of the stem-open MBs an excess (10 fold) of target DNA to the MB was used in the experiments described above. This excess was used to ensure that the interference of stem-closed MB is negligible. The target DNA concentration dependence on the MB opening equilibrium was investigated in detail and is described in the ESI, Fig. S3. $\dagger$ The MB showed a high sensitivity towards the target DNA. At equimolar concentrations of target and MB more than $50 \%$ of the MB opened to hybridize with the target. In control experiments using a non-complementary target sequence, no opening of the stem-closed MB was observable even at a large excess of noncomplementary target (see ESI, Fig. S4 $\dagger$ ).

\section{Conclusions}

We have designed a novel MB consisting of two fluorescent dyes (Alexa and RedX) (Scheme 2), which changes its fluorescence color upon binding to the complementary target DNA. Timeresolved fluorescence experiments showed efficient energy transfer from the donor (Alexa) to the acceptor $(\operatorname{RedX})$ in the stem-closed form. In the presence of the complementary target DNA the MB opened efficiently and energy transfer is blocked in the stem-open form (Scheme 1, right). This generates a fluorescence signature, which is clearly distinguishable from the fluorescence signature of the stem-closed form. The change in fluorescence signature rather than in fluorescence intensity, as in traditional dye-quencher MBs (Scheme 1, left), allows for ratiometric analysis of the fluorescence signal. Ratiometric analysis is generally more superior compared 
to pure intensity changes. ${ }^{9}$ Our MB showed a high response ratio of 10 upon addition of the complementary target DNA. Timeresolved fluorescence experiments showed that the response ratio of the $\mathrm{MB}$ can be improved by reducing the amount of the defective MB, where energy transfer is hindered in the absence of target. Traditionally, steady-state fluorescence spectroscopy is used almost exclusively for genetic analysis in conjunction with MBs. However, fluorescence lifetime and fluorescence depolarization measurements are more powerful analytical tools, which give additional information about the MB response to the target. ${ }^{25}$ This multi-technique approach should increase the reliability of MBs in genetic analysis.

\section{Acknowledgements}

This work was supported by the Center of Excellence in Genomic Science Grant P50 HG002806 from the National Institutes of Health and NSF CHE-04-15516. DLA thanks the NSF and DoDARO for support of this work, in part, through the following awards: (1) the NSF-IGERT Program, under Grant No. DGE9972892; (2) the NSF-MRSEC Program, under Grant No. DMR0213574; and (3) DoD-ARO, under Cooperative Agreement DAAD19-01-1-0759.

\section{References}

1 W. Tan, X. Fang, J. Li and X. Liu, Molecular beacons: a novel DNA probe for nucleic acid and protein studies, Chem.-Eur. J., 2000, 6, $1107-$ 1111.

2 T. Antony and V. Subramaniam, Molecular Beacons: Nucleic Acid Hybridization and Emerging Applications, J. Biomol. Struct. Dyn., 2001, 19, 4997-5004.

3 G. Bonnet, S. Tyagi, A. Libchaber and F. R. Kramer, Thermodynamic basis of the enhanced specificity of structured DNA probes, Proc. Natl. Acad. Sci. U. S. A., 1999, 96, 6171-6176.

4 S. Tyagi and F. R. Kramer, Molecular Beacons: Probes that Fluoresce upon Hybridization, Nat. Biotechnol., 1996, 14, 303-308.

5 S. Tyagi, D. P. Bratu and F. R. Kramer, Multicolor molecular beacons for allele discrimination, Nat. Biotechnol., 1998, 16, 49-58.

6 K. Faulds, L. Fruk, D. C. Robson, D. G. Thompson, A. Enright, W. E. Smith and D. Graham, A new approach for DNA detection by SERRS, Faraday Discuss., 2006, DOI: 10.1039/b506219e.

7 G. Bonnet, O. Krichevsky and A. Libchaber, Kinetics of conformational fluctuations in DNA hairpin-loops, Proc. Natl. Acad. Sci. U. S. A., 1998, 95, 8602-8606.

8 S. A. E. Marras, F. R. Kramer and S. Tyagi, Efficiencies of fluorescence resonance energy transfer and contact-mediated quenching in oligonucleotide probes, Nucleic Acids Res., 2002, 30, e122.
9 P. Zhang, T. Beck and W. Tan, Design of a Molecular Beacon DNA Probe with Two Fluorophores, Angew. Chem., Int. Ed., 2001, 40, 402405.

10 A. A. Martí, S. Jockusch, Z. Li, J. Ju and N. J. Turro, Molecular Beacons with intrinsically fluorescent nucleotides, Nucleic Acids Res., 2006, accepted, DOI: 10.1093/nar/gkl134.

$11 \mathrm{~K}$. Fujimoto, H. Shimizu and M. Inouye, Unambiguous Detection of Target DNAs by Excimer-Monomer Switching Molecular Beacons, J. Org. Chem., 2004, 69, 3271-3275.

12 C. J. Yang, S. Jockusch, M. Vincens, N. J. Turro and W. Tan, Lightswitching excimer probes for rapid protein monitoring in complex biological fluids, Proc. Natl. Acad. Sci. U. S. A., 2005, 102, 17278 17283 .

13 S. A. E. Marras, F. R. Kramer and S. Tyagi, Genotyping single nucleotide polymorphisms with molecular beacons, The Humana Press Inc., Towada, NJ, 2003, vol. 212, pp. 111-128.

14 J.-F. Brunet, E. Shapiro, S. A. Foster, E. R. Kandel and Y. Iino, Identification of a peptide specific for Aplysia sensory neurons by PCRbased differential screening, Science, 1991, 252, 856-859.

15 N. Panchuk-Voloshina, R. P. Haugland, J. Bishop-Stewart, M. K. Bhalgat, P. J. Millard, F. Mao, W.-Y. Leung and R. P. Haugland, Alexa dyes, a series of new fluorescent dyes that yield exceptionally bright, photostable conjugates, J. Histochem. Cytochem., 1999, 47, 1179-1188.

16 A. M. Iribarren, B. S. Sproat, P. Neuner, I. Sulston, U. Ryder and A. I. Lamond, 2'-O-alkyl oligoribonucleotides as antisense probes, Proc. Natl. Acad. Sci. U. S. A., 1990, 87, 7747-7751.

17 P. Bratu Diana, B.-J. Cha, M. Mhlanga Musa, R. Kramer Fred and S. Tyagi, Visualizing the distribution and transport of mRNAs in living cells, Proc. Natl. Acad. Sci. U. S. A., 2003, 100, 13308-13313.

18 A. I. Lamond and B. S. Sproat, Antisense oligonucleotide made of 2'O-AlkylRNA: their properties and applications in RNA biochemistry, FEBS Lett., 1993, 1-2, 123-127.

19 T. Heinlein, J.-P. Knemeyer, O. Piestert and M. Sauer, Photoinduced Electron Transfer between Fluorescent Dyes and Guanosine Residues in DNA-Hairpins, J. Phys. Chem. B, 2003, 107, 7957-7964.

20 K. Stoer, B. Haefner, O. Nolte, J. Wolfrum, M. Sauer and D.-P. Herten, Species-Specific Identification of Mycobacterial 16S rRNAS PCR Amplificons Using Smart Probes, Anal. Chem., 2005, 77, 71957203.

21 I. Nazarenko, R. Pires, B. Lowe, M. Obaidy and A. Rashtchian, Effect of primary and secondary structure of oligodeoxyribonucleotides on the fluorescent properties of conjugated dyes, Nucleic Acids Res., 2002, 30, 2089-2195.

22 E. Rusinova, V. Tretyachenko-Ladokhina, O. E. Vele, D. F. Senear and J. B. A. Ross, Alexa and Oregon Green dyes as fluorescence anisotropy probes for measuring protein-protein and protein-nucleic acid interactions, Anal. Biochem., 2002, 308, 18-25.

23 R. A. Hochstrasser, S.-M. Chen and D. P. Millar, Distance distribution in a dye-linked oligonucleotide determined by time-resolved fluorescence energy transfer, Biophys. Chem., 1992, 45, 133-141.

24 J. R. Lakowicz, Topics in Fluorescence Spectroscopy, Volume 5: Nonlinear and Two-Photon-Induced Fluorescence, Plenum Press, New York, 1997, vol. 5, 544 pp.

25 A. Tsourkas, M. A. Behlke, Y. Xu and G. Bao, Spectroscopic features of dual fluorescence/luminescence resonance energy-transfer molecular beacons, Anal. Chem., 2003, 75, 3697-3703. 